\title{
Chemical Mapping of Block Copolymer Electrolytes by EFTEM Spectrum Imaging
}

\author{
F.I. Allen,* M. Watanabe, ${ }^{* *}$ N.P. Balsara, ${ }^{* * *}$ and A.M. Minor* \\ * National Center for Electron Microscopy, Lawrence Berkeley National Laboratory, CA 94720- \\ 8250, and Department of Materials Science and Engineering, University of California, Berkeley, CA \\ 94720-1760 \\ ** Department of Materials Science and Engineering, Lehigh University, Bethlehem, PA 18015 \\ *** Materials Science Division, Lawrence Berkeley National Laboratory, CA 94720-8250, and \\ Department of Chemical Engineering, University of California, Berkeley, CA 94720-1462
}

We investigate the spatial distribution of chemical phases in dry block copolymer electrolytes. The constituent blocks of these copolymers can be chosen to produce a material which combines the conductivity of soft nanoscale channels with the rigidity of a non-conducting glassy matrix. Hence block copolymer electrolytes are favorable candidates for the solid electrolytes in energy-technology devices such as fuel cells and solid state batteries [1]. By imaging the distribution of ionic species in block copolymer electrolytes we aim to increase our understanding of the mechanism of ionic conductivity in these materials.

In this work we focus on lamellar poly(styrene-block-ethylene oxide) (PS-PEO). The PEO phase provides the conductive channels and ionic species introduced into the system co-ordinate with the ethylene oxide groups of the PEO block. Thin films $(<100 \mathrm{~nm})$ of PS-PEO electrolyte have been prepared by spin casting from a solution of the polymer and the salt sodium hexafluorophosphate $\left(\mathrm{NaPF}_{6}\right)$ onto holey silicon nitride membranes. The samples are then annealed at $90^{\circ} \mathrm{C}$. A brightfield TEM micrograph showing the microphase structure of the PS-PEO film supported across a hole in the silicon nitride membrane is presented in Figure 1. The mass fraction of sodium ions in the block copolymer is of the order of $1 \%$.

The chemical phases in the block copolymer electrolyte are distinguished by low-loss energy filtered TEM spectrum imaging (EFTEM SI). This approach involves the acquisition of a series of images at adjacent energy positions in the plasmon peak range of the electron energy loss spectrum using a small energy-selecting slit. The plasmon peak is characteristic of the chemical composition of the sample and is far more intense than the core loss edges used for conventional elemental mapping. Thus low-loss EFTEM SI presents a convenient method for the quantitative analysis of radiation sensitive materials such as polymers.

For our EFTEM SI measurements we employ a 200kV monochromated Zeiss Libra microscope. Typically the EFTEM slices are recorded from $2-60 \mathrm{eV}$ in $1 \mathrm{eV}$ steps using an energy slit of $5 \mathrm{eV}$. In order to distinguish the chemical phases of the specimen we apply multivariate statistical analysis (MSA) to the EFTEM SI data [2]. This allows us to identify the principal components of the EFTEM SI and to reconstruct a noise-reduced dataset. The plasmon peaks corresponding to the identified phases are then extracted and used for MLLS fitting. Using this technique we have identified three main phases in the PS-PEO electrolyte. Figures 2a, 2b, and 2c show the MLLS fits for the first (PS), second (PEO) and third phase, respectively. The combined RGB image is shown in Figure $2 \mathrm{~d}$. We propose that the third phase, which is located at the edge of the PEO domains, gives the spatial distribution of salt ions in the block copolymer. 


\section{References}

[1] J.M. Tarascon and M. Armand, Nature 414 (2001) 359.

[2] M. Watanabe et al., Microsc. Anal. 23(7) (2009) 5.

[3] The authors acknowledge the support of the U.S. Department of Energy, Office of Basic Energy Sciences, Division of Materials Sciences and Engineering under Contract No. DEAC02-05CH11231.

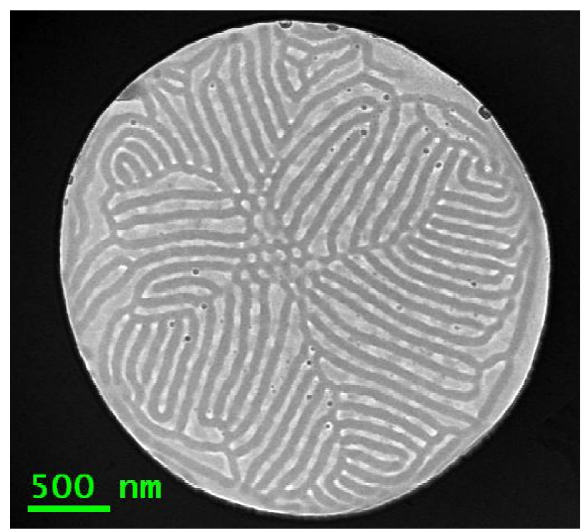

FIG. 1. Bright-field TEM micrograph of a thin film of PS-PEO spin cast over holey silicon nitride.

a

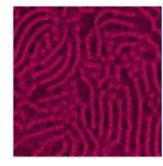

b

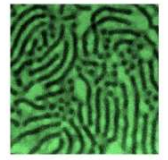

C

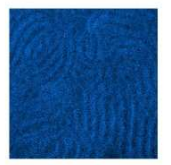

d

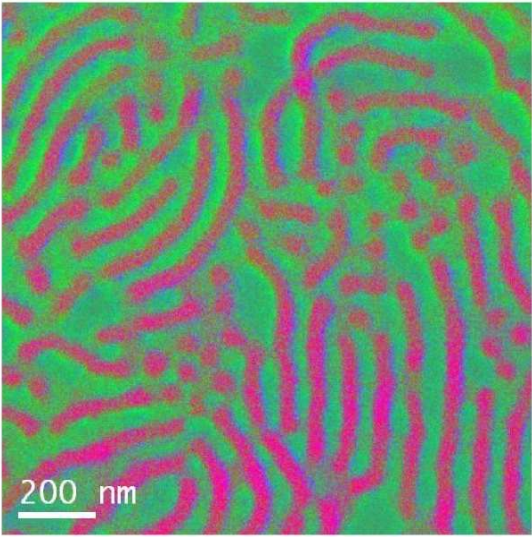

FIG. 2. Colored MLLS fits corresponding to phase 1 (PS), phase 2 (PEO) and phase 3 shown in (a), (b), and (c), respectively. Combined RGB image shown in (d). 